\title{
Prevalence of and factors associated with non- communicable diseases among Bangladeshi adults: investigation from nationally surveyed data
}

Md. Ashfikur Rahman ( $\square$ ashfikur@ku.ac.bd )

Khulna University

Henry Ratul Halder

University of Manitoba

Satyajit Kundu

Southeast University

Md. Hasan Al Banna

Patuakhali Science and Technology

\section{Research Article}

Keywords: Non-communicable disease, diabetes, hypertension, co-morbidity, BDHS

Posted Date: November 30th, 2021

DOl: https://doi.org/10.21203/rs.3.rs-1103907/v1

License: (c) (i) This work is licensed under a Creative Commons Attribution 4.0 International License. Read Full License 


\section{Abstract \\ Background}

Chronic non-communicable diseases, owing to their increasing prevalence, are the greatest constraint to disease burden reduction in Bangladesh. As a result, we concentrated on determining the prevalence and risk factors for major chronic non-communicable diseases (NCDs) among adult Bangladeshis.

\section{Methods}

Data from Bangladesh Demographic and Health Survey (BDHS) 2017-18 were analyzed. If a participant had diabetes or hypertension, it was classified as NCD. Whereas comorbidity is defined as a subject having both diabetes and hypertension. Both the unadjusted and adjusted log-binomial regression models considering the survey weights were employed to identify the factors associated with NCDs and comorbidity.

\section{Results}

The overall prevalence (age-adjusted) of NCDs (40.43\% (95\% Cl: 40.29-40.56) diabetes and hypertension was 11.55\% (95\% Cl: 11.46-11.64) and 35.04\% (95\% Cl: 34.91-35.17), respectively, while 6.16\% (95\% Cl: 6.09-6.23) of participants had comorbidity. The adjusted regression model shows that being aged $>34$ years, and overweight or obese were significant risk factors of all NCDs, where being involved in work and from rich households were found as risk factors of diabetes and comorbidity. Smoker participants and females were more likely to have hypertension compared to their counterparts. Contrary, being underweight was a protective factor of having NCDs, similarly, engage in work was found as protective factors of diabetes and co-morbidity.

\section{Conclusion}

A growing prevalence of diabetes, hypertension, and comorbidity was discovered in this study. To reduce the burden of these NCDs, it is necessary to take the necessary steps.

\section{Background}

The major threat to public health in this twenty-first century worldwide remained non-communicable diseases (NCDs), leading to ill health, death and disability, economic loss, life loss, declining living standards, and poor social development in both high-income to low-income countries (LMICs). NCDs are predicted to kill 41 million people each year, accounting for $71 \%$ of all deaths worldwide, with $77 \%$ of those deaths occurring in LMICs including Bangladesh [1]. In Bangladesh various infectious diseases are still prevalent and they have long been the most prominent contributors to disease burden, but the burden of NCDs is also escalating [2,3] which is an ultimate threat to fulfil the country's attainment of Sustainable Development Goal (SDG) 3.4, which calls for $30 \%$ deterioration in NCD-related mortality in the Global Action Plan for the Prevention and Control of NCDs through "prevention and treatment and promote mental health and well-being" [4, 5]. 
Bangladesh is undergoing a demographic and epidemiological transition, and it is currently dealing with a double burden of diseases [6]. In recent decades, life expectancy has risen significantly and fertility rate declined due to socioeconomic well-being, resulting in a significant burden of NCDs $[7,8]$. In Bangladesh, $67 \%$ of annual deaths are attributable to NCDs which has been projected to be augmented further in coming decades [9]. Several recent population-based studies in Bangladesh have revealed an unprecedented upsurge in the prevalence of NCDs, compared to prior population-based research $[3,10,11]$. Recent Bangladesh Demographic and Health Survey (BDHS 2017-18) also portrays same increment of different NCDs diseases such as hypertension, diabetes and obesity [12-15]. Long-term care and treatment programmes for NCDs frequently demand a substantial financial investment, and poor individuals have limited access to such treatments, even when they are accessible [7]. Although, Bangladesh has already outperformed in achieving Millennium Development Goals (MDGs) particularly in child and maternal health issues but to combat against NCDs burden government needs to pay high priority devotion.

Multiple causes are associated with developing NCDs, including nutritional transition, rapid urbanization, changing lifestyle and behavioural factors (i.e., use of tobacco \& alcohol, high intake of rich foods, extra screen timing with mobile, laptop, internet, low intake of vegetables, and fruits, physical inactivity) along with some metabolic factors (such as for overweight, obesity, blood sugar, blood pressure, and high cholesterol level) and limited access to proper health care facilities [16-19]. Prevention efforts inside the healthcare system must adopt a wider public health strategy to encourage individuals to change their unhealthy lifestyles, which contribute to the development of NCDs. Data on the prevalence and distribution of NCD risk factors among adults can aid in predicting the future burden of NCDs and developing evidence-based measures to prevent the emergence of new NCDs. However, the lack of established vital statistics systems and trustworthy population-level data in Bangladesh limits our understanding of the epidemiology of NCDs and its recent upsurge.

In line with UN and WHO recognition for the growing challenges posed by the NCDs, health systems need to be equipped to deal with changing disease burden patterns; however, Bangladesh does not have the suitable arrangements to assist achieve its goals, in alignment with the NCD policy indicators outlined in the action plan. Recognizing these aspects, we carried out a cross-sectional study using the recent round population-based survey data to estimate the prevalence and factors associated with non-communicable diseases among Bangladeshi adult population which will aid relevant stakeholders in developing appropriate prevention strategies for the certain population affected by NCDs.

\section{Methods}

\section{Data sources}

The most recent 2017-18 BDHS dataset was used in the research. The National Institute of Population Research and Training, Medical Education and Family Welfare Division, and the Ministry of Health and Family Welfare conducted the survey from October 2017 to March 2018. The main goal of the survey was to evaluate health indicators and offer an overview of population, maternal and child health, and the status of numerous NCDs as hypertension and diabetes.

\section{Study population and survey design}


The sampling frame used for the 2017-18 BDHS is the complete list of enumeration areas (EAs) covering the entire population of Bangladesh. The survey used a list of enumeration areas (EAs) provided by the Bangladesh Bureau of Statistics of the 2011 Population and Housing Census of the People's Republic of Bangladesh. The survey's primary sampling unit (PSU) is an EA covering on average 120 households in 2017-18. The 2017-18 BDHS was a multistage stratified cluster sample of households' survey, carried out in two and three stages in rural and urban settings. In the first sampling stage, rural wards were selected, following PSUs, and then households were selected from PSUs. In urban areas, wards were selected through the PSUs technique, and one EA was selected from each PSU. Then, the households were chosen from the selected EAs sample. A detailed description of the survey design, methodologies, sample size, questionnaires, and findings are available in the final summary report of 2017-18 BDHS. Anthropometry and BP were also systematically measured from the selected subsample of 2017-18 BDHS[12]. A total of 12, 152 unweighted sample was found in the original dataset, which, after giving weight increased to 12,975 . However, we had to exclude 8,82 cases due to missing values in some of the variables we considered in our analysis, making the weighted number of observations 12,093 for our analysis.

\section{Dependent variable}

Participants with the fasting blood glucose (FBG) value $\geq 7.0 \mathrm{mmol} / \mathrm{l}(126 \mathrm{mg} / \mathrm{dl})$ and those who were taking medications to lower their elevated blood glucose levels were classified as diabetic[20]. For hypertension, the ACC/AHA 2017 guideline has been used. Briefly, a participant with systolic blood pressure (SBP) of $\geq 130 \mathrm{mmHg}$ and/or diastolic blood pressure (DBP) of $\geq 80 \mathrm{mmHg}$ or who were taking any prescribed antihypertensive drugs to control blood pressure (BP) was categorized as hypertensive [2]. The dependent variable for this study was hypertension. Trained health technicians measured BP three times using LIFE SOURCEß UA-767 Plus BP monitor at about ten minutes interval[12]. Then, the average of second and third measurements was used to report respondents' final BP[12].

While according to the ACC/AHA 2017 guideline, individuals with a SBP of $\geq 130 \mathrm{mmHg}$ and/or a DBP of $\geq 80 \mathrm{mmHg}$ or who were taking any prescribed antihypertensive drugs to control BP were categorized as hypertensive[21]. The category of prehypertension was transformed into elevated blood pressure in the 2017 ACC/AHA guideline[21]. This study also investigated the NCD and comorbidity status of the participants. If a participant suffered from either diabetes or hypertension, then it was recorded as NCD. Whereas, if a participant had both diabetes and hypertension, then it is considered as comorbidity.

\section{Explanatory variables}

The explanatory variables included in the study were selected based on previous literature reporting the risk of hypertension in LMICs setting [17, 22-27]. The household factors included administrative divisions (Barisal, Chittagong, Dhaka, Khulna, Rajshahi, Rangpur, Sylhet, Mymensingh); place of residence (urban, rural); and wealth status (poorest, poorer, middle, richer, richest), whereas the socioeconomic and individual factors included: age of the participants (18-24, 25-34, 35-44, 45-54, 55-64, $\geq 65$ ); sex of the participants (male, female); education level (no education, primary, secondary, higher); and occupational status (not working, working). Behavioral characteristics included smoking habit (no, yes) and body mass index (BMI) level. We have used global cut-off points for BMI classification: underweight $\left(<18.5 \mathrm{~kg} / \mathrm{m}^{2}\right)$, normal $\left(18.5-25.0 \mathrm{~kg} / \mathrm{m}^{2}\right)$, overweight $\left(25.1-29.9 \mathrm{~kg} / \mathrm{m}^{2}\right)$, and obese $\left(\geq 30.0 \mathrm{~kg} / \mathrm{m}^{2}\right)[28]$.

\section{Statistical analysis}


Considering the complex survey of BDHS, we prepared the data using the survey weights before the analysis. Next, the normality assumption of continuous variables was investigated from their distribution, and it was reported in the paper with medians and interquartile ranges (IQRs). Then, we estimated the prevalence of hypertension and reported the differences between the two guidelines. We reported the prevalence of hypertension by background characteristics accounting for complex survey design/survey weight. It is notable to mention that survey weights only account for the sampling scheme. Therefore, we standardized the prevalence of hypertension for the same standard population to remove or minimize the impact of differences in participant's age and sex distribution. On the next step, we fitted the selected explanatory variables in unadjusted log-binomial regression model. Thereafter, we executed a log-binomial regression model considering survey weights including the explanatory variables having p-values $(<0.05)$ in the unadjusted analysis to identify the factors associated with hypertension, describing results with prevalence ratios (PRs) and their 95\% confidence intervals (Cls) and p-values. We used Statistical Package for Social Science (SPSS) version 26 and SAS® OnDemand for Academics for data analysis.

\section{Results}

Sociodemographic characteristics of study participants are presented in Table 1. Of a total of 12093 respondents, more than half $(57.2 \%)$ of them were female. Two-thirds of the respondents (64.9\%) were aged between 18 to 44 years old with a median age of 21.9 years. Approximately, eighteen percent of the participants were underweighted and a quarter of them (24.1\%) were either overweight or obese. More than one-third (39.1\%) of the respondents' wealth status was poor, and majority of them (60.9\%) were involved in any type of work for their livelihood. 
Table 1

Background characteristics of the study participants $(\mathrm{N}=12,093)$

\begin{tabular}{|c|c|c|c|c|c|}
\hline $\begin{array}{l}\text { Background } \\
\text { Characteristics }\end{array}$ & $\begin{array}{l}\text { Total } \\
(\mathrm{N}=12,093)\end{array}$ & $\begin{array}{l}\text { NCD } \\
(n=3,986)\end{array}$ & $\begin{array}{l}\text { Diabetes } \\
(n=1,201)\end{array}$ & $\begin{array}{l}\text { Hypertension } \\
(n=3,338)\end{array}$ & $\begin{array}{l}\text { Comorbidity } \\
(n=553)\end{array}$ \\
\hline \multicolumn{6}{|c|}{ Geographic Location, n (\%) } \\
\hline Barisal & $666(5.5)$ & $254(6.3)$ & $64(5.4)$ & $216(6.5)$ & $28(5.1)$ \\
\hline Chittagong & $2082(17.2)$ & 734 (18.4) & 232 (19.4) & $628(18.8)$ & $126(22.8)$ \\
\hline Dhaka & $2791(23.1)$ & $906(22.7)$ & 399 (33.2) & $664(19.9)$ & $157(28.4)$ \\
\hline Khulna & $1511(12.5)$ & $505(12.7)$ & $125(10.4)$ & $454(13.6)$ & $74(13.3)$ \\
\hline Mymensingh & $988(8.2)$ & $275(6.9)$ & $78(6.5)$ & $230(6.9)$ & $33(5.9)$ \\
\hline Rajshahi & $1751(14.5)$ & $563(14.1)$ & $141(11.7)$ & $482(14.4)$ & $60(10.9)$ \\
\hline Rangpur & 1515 (12.5) & $504(12.7)$ & $85(7.0)$ & $461(13.8)$ & $41(7.5)$ \\
\hline Sylhet & $789(6.5)$ & $245(6.2)$ & $77(6.4)$ & $203(6.1)$ & $34(6.2)$ \\
\hline \multicolumn{6}{|l|}{ Place of Residence, $n(\%)$} \\
\hline Urban & $3208(26.5)$ & $1151(28.9)$ & $424(35.3)$ & $921(27.6)$ & $194(35.2)$ \\
\hline Rural & 8885 (73.5) & $2835(71.1)$ & 777 (64.7) & 2417 (72.4) & $359(64.8)$ \\
\hline \multicolumn{6}{|l|}{ Participant's Sex, n (\%) } \\
\hline Male & $5178(42.8)$ & $1680(42.1)$ & $544(45.3)$ & $1367(41.0)$ & 231 (41.9) \\
\hline Female & $6915(57.2)$ & $2306(57.9)$ & $657(54.7)$ & $1971(59.0)$ & $322(58.1)$ \\
\hline \multicolumn{6}{|c|}{$\begin{array}{l}\text { Participant's Age (Years), n } \\
\text { (\%) }\end{array}$} \\
\hline Median (IQR) & $36(26-50)$ & $47(35-60)$ & $46(35-59)$ & $48(36-60)$ & $52(40-62)$ \\
\hline $18-34$ & $5413(44.8)$ & 905 (22.7) & $284(23.7)$ & $680(20.4)$ & $60(10.9)$ \\
\hline $35-44$ & $2429(20.1)$ & $862(21.6)$ & $271(22.6)$ & 701 (21.0) & 110 (19.9) \\
\hline $45-54$ & 1685 (13.9) & $766(19.2)$ & $250(20.8)$ & 653 (19.6) & $136(24.6)$ \\
\hline $55-64$ & 1362 (11.3) & 725 (18.2) & 215 (17.9) & $640(19.2)$ & $130(23.5)$ \\
\hline$>=65$ & $1204(10.0)$ & $728(18.3)$ & $181(15.1)$ & $664(19.9)$ & $117(21.1)$ \\
\hline \multicolumn{6}{|l|}{ BMI Level ${ }^{\mathbb{Q}}, \mathrm{n}(\%)$} \\
\hline Median (IQR) & $\begin{array}{l}21.9(19.4- \\
24.9)\end{array}$ & $\begin{array}{l}23.4(20.4- \\
26.3)\end{array}$ & $\begin{array}{l}23.8(20.8- \\
26.9)\end{array}$ & $\begin{array}{l}23.6(20.6- \\
26.4)\end{array}$ & $\begin{array}{l}25.0(22.2- \\
27.5)\end{array}$ \\
\hline $\begin{array}{l}\text { Underweight }(<18.5 \\
\left.\mathrm{kg} / \mathrm{m}^{2}\right)\end{array}$ & 2067 (17.3) & $461(11.8)$ & $128(10.9)$ & 365 (11.2) & $32(5.9)$ \\
\hline $\begin{array}{l}\text { Normal }(18.5-24.9 \\
\left.\mathrm{kg} / \mathrm{m}^{2}\right)\end{array}$ & 7014 (58.7) & $2045(52.2)$ & 606 (51.2) & 1683 (51.5) & $244(45.4)$ \\
\hline
\end{tabular}




\begin{tabular}{|c|c|c|c|c|c|}
\hline $\begin{array}{l}\text { Background } \\
\text { Characteristics }\end{array}$ & $\begin{array}{l}\text { Total } \\
(\mathrm{N}=12,093)\end{array}$ & $\begin{array}{l}\text { NCD } \\
(n=3,986)\end{array}$ & $\begin{array}{l}\text { Diabetes } \\
(n=1,201)\end{array}$ & $\begin{array}{l}\text { Hypertension } \\
(n=3,338)\end{array}$ & $\begin{array}{l}\text { Comorbidity } \\
(n=553)\end{array}$ \\
\hline $\begin{array}{l}\text { Overweight (25.0-29.9 } \\
\left.\mathrm{kg} / \mathrm{m}^{2}\right)\end{array}$ & $2389(20.0)$ & $1159(29.6)$ & $353(29.9)$ & $1004(30.7)$ & $199(37.1)$ \\
\hline Obesity $\left(\geq 30.0 \mathrm{~kg} / \mathrm{m}^{2}\right)$ & $489(4.1)$ & $251(6.4)$ & $96(8.0)$ & $219(6.7)$ & $63(11.6)$ \\
\hline \multicolumn{6}{|l|}{ Education Level, n (\%) } \\
\hline No Education & $3114(25.8)$ & $1290(32.4)$ & $307(25.5)$ & $1140(34.1)$ & $156(28.2)$ \\
\hline Primary & $3618(29.9)$ & $1175(29.5)$ & 370 (30.9) & $958(28.7)$ & $153(27.8)$ \\
\hline Secondary & $3562(29.5)$ & $1012(25.4)$ & $345(28.7)$ & $830(24.9)$ & $163(29.4)$ \\
\hline Higher & $1799(14.9)$ & $509(12.8)$ & 179 (14.9) & $410(12.3)$ & $81(14.6)$ \\
\hline \multicolumn{6}{|l|}{ Occupational Status, n (\%) } \\
\hline Not Working & $4732(39.1)$ & $1754(44.0)$ & $560(46.6)$ & $1476(44.2)$ & $282(50.9)$ \\
\hline Working & $7361(60.9)$ & $2232(56.0)$ & $641(53.4)$ & $1862(55.8)$ & $271(49.1)$ \\
\hline \multicolumn{6}{|l|}{ Wealth Status, n (\%) } \\
\hline Poor & $4729(39.1)$ & $1338(33.6)$ & $277(23.1)$ & $1161(34.8)$ & $100(18.0)$ \\
\hline Middle & $2494(20.6)$ & 780 (19.6) & $199(16.5)$ & $672(20.1)$ & $90(16.3)$ \\
\hline Rich & $4870(40.3)$ & $1868(46.9)$ & $725(60.4)$ & $1505(45.1)$ & $363(65.6)$ \\
\hline \multicolumn{6}{|l|}{ Smoking Status", n (\%) } \\
\hline No & $10380(85.9)$ & 3340 (83.9) & $1019(85.0)$ & 2792 (83.7) & $470(85.1)$ \\
\hline Yes & $1709(14.1)$ & $643(16.1)$ & $180(15.0)$ & $546(16.3)$ & $83(14.9)$ \\
\hline \multicolumn{6}{|l|}{$\begin{array}{l}\text { Source of Drinking Water, } n \\
\text { (\%) }\end{array}$} \\
\hline Improved & $11886(98.3)$ & 3929 (98.6) & $1190(99.1)$ & 3287 (98.5) & $547(99.0)$ \\
\hline Unimproved & 207 (1.7) & $57(1.4)$ & $11(0.9)$ & $51(1.5)$ & $6(1.0)$ \\
\hline \multicolumn{6}{|l|}{ Sanitation Facility, n (\%) } \\
\hline Improved & 8017 (66.3) & $2805(70.4)$ & $887(73.9)$ & $2350(70.4)$ & $432(78.1)$ \\
\hline Unimproved & 3901 (32.3) & $1124(28.2)$ & $302(25.2)$ & $937(28.1)$ & $115(20.9)$ \\
\hline Open defecation & $175(1.4)$ & $57(1.4)$ & $11(0.9)$ & $51(1.5)$ & $6(1.0)$ \\
\hline
\end{tabular}




\begin{tabular}{|llllll|}
\hline Background & Total & NCD & Diabetes & Hypertension & Comorbidity \\
Characteristics & $(N=12,093)$ & $(n=3,986)$ & $(n=1,201)$ & $(n=3,338)$ & $(n=553)$ \\
\hline
\end{tabular}

${ }^{{ }_{B}} \mathrm{BMI}$ level had $134,70,18,67$, and 15 observations missing for total, NCD, diabetic, hypertensive, and comorbid participants, respectively.

*Smoking status had 4, 3, and 2 missing observations for total, NCD, and diabetic participants, respectively.

'Indicates no sanitation facility or open-air defecation.

NCD: Non-communicable disease; IQR: Inter-quartile range; BMI: Body mass index.

\section{Prevalence of non-communicable diseases (NCDs)}

The age-adjusted prevalence of NCD was 40.4\% (95\% Cl: 40.29-40.56) among Bangladeshi adults. Specifically, the age-adjusted prevalence of DM and HT was 11.55\% (95\% Cl: 11.46-11.64) and 35.04\% (95\% Cl: 34.91-35.17), respectively among the study participants. About $6.16 \%$ (95\% Cl: $6.09-6.23)$ of the study participants showed comorbidity (co-existence of DM and HT) (Table 2). 
Table 2

Age-standardized prevalence of non-communicable diseases with 95\% $\mathrm{Cl}(\mathrm{N}=12,093)$

\begin{tabular}{|c|c|c|c|c|}
\hline Independent & NCD & Diabetes & Hypertension & Comorbidity \\
\hline Variables & $\begin{array}{l}\text { Prevalence }(95 \% \\
\mathrm{Cl})\end{array}$ & $\begin{array}{l}\text { Prevalence } \\
(95 \% \mathrm{Cl})\end{array}$ & $\begin{array}{l}\text { Prevalence } \\
(95 \% \mathrm{Cl})\end{array}$ & $\begin{array}{l}\text { Prevalence } \\
(95 \% \mathrm{Cl})\end{array}$ \\
\hline Overall Prevalence & $\begin{array}{l}40.43(40.29- \\
40.56)\end{array}$ & $\begin{array}{l}11.55(11.46- \\
11.64)\end{array}$ & $\begin{array}{l}35.04(34.91- \\
35.17)\end{array}$ & $6.16(6.09-6.23)$ \\
\hline \multicolumn{5}{|c|}{ Geographic Location } \\
\hline Barisal & $\begin{array}{l}45.93(45.50- \\
46.35)\end{array}$ & $\begin{array}{l}11.73(11.46- \\
12.01)\end{array}$ & $\begin{array}{l}40.35(39.93- \\
40.77)\end{array}$ & $6.15(5.95-6.36)$ \\
\hline Chittagong & $\begin{array}{l}43.03(42.65- \\
43.42)\end{array}$ & $\begin{array}{l}13.84(13.58- \\
14.11)\end{array}$ & $\begin{array}{l}37.09(36.72- \\
37.46)\end{array}$ & $7.90(7.69-8.11)$ \\
\hline Dhaka & $\begin{array}{l}40.16(39.77- \\
40.55)\end{array}$ & $\begin{array}{l}17.20(16.90- \\
17.51)\end{array}$ & $\begin{array}{l}30.58(30.22- \\
30.95)\end{array}$ & $7.62(7.42-7.84)$ \\
\hline Khulna & $\begin{array}{l}41.42(41.05- \\
41.78)\end{array}$ & $\begin{array}{l}11.06(10.83- \\
11.30)\end{array}$ & $\begin{array}{l}37.43(37.07- \\
37.79)\end{array}$ & 7.08 (6.89-7.27) \\
\hline Mymensingh & $\begin{array}{l}34.86(34.47- \\
35.25)\end{array}$ & $8.97(8.74-9.21)$ & $\begin{array}{l}29.82(29.44- \\
30.19)\end{array}$ & $3.93(3.77-4.09)$ \\
\hline Rajshahi & $\begin{array}{l}39.42(39.04- \\
39.80)\end{array}$ & $\begin{array}{l}10.53(10.30- \\
10.77)\end{array}$ & $\begin{array}{l}34.40(34.03- \\
34.77)\end{array}$ & $5.51(5.34-5.69)$ \\
\hline Rangpur & $\begin{array}{l}39.77 \text { (39.39- } \\
40.15)\end{array}$ & 7.29 (7.09-7.49) & $\begin{array}{l}36.75(36.38- \\
37.13)\end{array}$ & $4.28(4.12-4.44)$ \\
\hline Sylhet & $\begin{array}{l}38.85(38.45- \\
39.26)\end{array}$ & $\begin{array}{l}11.83(11.57- \\
12.11)\end{array}$ & $\begin{array}{l}33.62 \text { (33.23- } \\
34.02)\end{array}$ & $6.61(6.40-6.82)$ \\
\hline \multicolumn{5}{|l|}{ Place of Residence } \\
\hline Urban & $\begin{array}{l}43.45(43.21- \\
43.68)\end{array}$ & $\begin{array}{l}14.66(14.49- \\
14.83)\end{array}$ & $\begin{array}{l}37.10(36.87- \\
37.33)\end{array}$ & $8.31(8.18-8.44)$ \\
\hline Rural & $\begin{array}{l}38.83(38.66- \\
39.00)\end{array}$ & $\begin{array}{l}9.90(9.80- \\
10.01)\end{array}$ & $\begin{array}{l}33.96 \text { (33.79- } \\
34.12)\end{array}$ & $5.03(4.95-5.11)$ \\
\hline \multicolumn{5}{|l|}{ Participant's Sex } \\
\hline Male & $\begin{array}{l}38.56(38.35- \\
38.76)\end{array}$ & $\begin{array}{l}11.52(11.39- \\
11.66)\end{array}$ & $\begin{array}{l}32.68 \text { (32.49- } \\
32.88)\end{array}$ & $5.65(5.55-5.75)$ \\
\hline Female & $\begin{array}{l}41.96(41.78- \\
42.15)\end{array}$ & $\begin{array}{l}11.56(11.44- \\
11.69)\end{array}$ & $\begin{array}{l}36.98(36.80- \\
37.16)\end{array}$ & $6.58(6.49-6.68)$ \\
\hline \multicolumn{5}{|c|}{ Participant's Age (Years) } \\
\hline $18-34$ & $17.87(17.67,18.08)$ & $5.52(5.40-5.64)$ & $\begin{array}{l}13.75(13.57- \\
13.93)\end{array}$ & $1.40(1.34-1.46)$ \\
\hline $35-44$ & $35.88(35.57,36.18)$ & $\begin{array}{l}10.93(10.73- \\
11.13)\end{array}$ & $\begin{array}{l}29.56(29.27- \\
29.85)\end{array}$ & $4.61(4.47-4.74)$ \\
\hline $45-54$ & $45.8(45.47,46.14)$ & $\begin{array}{l}14.43(14.20- \\
14.67)\end{array}$ & $\begin{array}{l}39.36(39.03- \\
39.70)\end{array}$ & $7.99(7.81-8.18)$ \\
\hline
\end{tabular}




\begin{tabular}{|c|c|c|c|c|}
\hline Independent & NCD & Diabetes & Hypertension & Comorbidity \\
\hline Variables & $\begin{array}{l}\text { Prevalence }(95 \% \\
\mathrm{Cl})\end{array}$ & $\begin{array}{l}\text { Prevalence } \\
(95 \% \mathrm{Cl})\end{array}$ & $\begin{array}{l}\text { Prevalence } \\
(95 \% \mathrm{Cl})\end{array}$ & $\begin{array}{l}\text { Prevalence } \\
(95 \% \mathrm{Cl})\end{array}$ \\
\hline $55-64$ & $54.53(54.19,54.87)$ & $\begin{array}{l}15.86(15.61- \\
16.11)\end{array}$ & $\begin{array}{l}48.45(48.11- \\
48.79)\end{array}$ & $9.78(9.58-9.98)$ \\
\hline$>=65$ & $62.14(61.83,62.46)$ & $\begin{array}{l}14.89(14.66- \\
15.13)\end{array}$ & $\begin{array}{l}57.41(57.09- \\
57.74)\end{array}$ & $\begin{array}{l}10.16(9.97- \\
10.36)\end{array}$ \\
\hline \multicolumn{5}{|l|}{ BMI Level } \\
\hline $\begin{array}{l}\text { Underweight }(<18.5 \\
\left.\mathrm{kg} / \mathrm{m}^{2}\right)\end{array}$ & $\begin{array}{l}29.21(28.90- \\
29.51)\end{array}$ & $6.29(6.13-6.45)$ & $\begin{array}{l}24.86(24.58- \\
25.15)\end{array}$ & $1.94(1.86-2.04)$ \\
\hline $\begin{array}{l}\text { Normal }(18.5-24.9 \\
\left.\mathrm{kg} / \mathrm{m}^{2}\right)\end{array}$ & $\begin{array}{l}37.74(37.56- \\
37.92)\end{array}$ & $\begin{array}{l}10.37(10.26- \\
10.48)\end{array}$ & $\begin{array}{l}32.47(32.30- \\
32.65)\end{array}$ & $5.10(5.02-5.18)$ \\
\hline $\begin{array}{l}\text { Overweight (25.0-29.9 } \\
\left.\mathrm{kg} / \mathrm{m}^{2}\right)\end{array}$ & $\begin{array}{l}54.76(54.45- \\
55.08)\end{array}$ & $\begin{array}{l}17.43(17.19- \\
17.67)\end{array}$ & $\begin{array}{l}48.39(48.08- \\
48.71)\end{array}$ & $\begin{array}{l}11.06(10.86- \\
11.26)\end{array}$ \\
\hline Obesity $\left(\geq 30.0 \mathrm{~kg} / \mathrm{m}^{2}\right)$ & $\begin{array}{l}58.76(58.08- \\
59.44)\end{array}$ & $\begin{array}{l}22.98(22.40- \\
23.56)\end{array}$ & $\begin{array}{l}51.90(51.21- \\
52.59)\end{array}$ & $\begin{array}{l}16.11(15.61- \\
16.63)\end{array}$ \\
\hline \multicolumn{5}{|l|}{ Education Level } \\
\hline No Education & $\begin{array}{l}45.88(45.64- \\
46.13)\end{array}$ & $\begin{array}{l}10.27(10.12- \\
10.42)\end{array}$ & $\begin{array}{l}41.42(41.18- \\
41.66)\end{array}$ & $5.80(5.69-5.92)$ \\
\hline Primary & $\begin{array}{l}39.26(39.01- \\
39.51)\end{array}$ & $\begin{array}{l}11.40(11.24- \\
11.56)\end{array}$ & $\begin{array}{l}33.38(33.14- \\
33.62)\end{array}$ & $5.52(5.40-5.64)$ \\
\hline Secondary & $\begin{array}{l}36.23(35.96- \\
36.50)\end{array}$ & $\begin{array}{l}12.48(12.29- \\
12.67)\end{array}$ & $\begin{array}{l}30.63(30.37- \\
30.89)\end{array}$ & $6.88(6.73-7.02)$ \\
\hline Higher & $\begin{array}{l}37.32(36.95- \\
37.70)\end{array}$ & $\begin{array}{l}13.35(13.09- \\
13.61)\end{array}$ & $\begin{array}{l}31.18(30.82- \\
31.55)\end{array}$ & $7.21(7.01-7.41)$ \\
\hline \multicolumn{5}{|l|}{ Occupational Status } \\
\hline Not Working & $\begin{array}{l}48.10(47.88- \\
48.32)\end{array}$ & $\begin{array}{l}14.03(13.87- \\
14.18)\end{array}$ & $\begin{array}{l}42.41(42.19- \\
42.63)\end{array}$ & $8.34(8.22-8.46)$ \\
\hline Working & $\begin{array}{l}35.24(35.07- \\
35.42)\end{array}$ & $9.87(9.76-9.98)$ & $\begin{array}{l}30.06(29.90- \\
30.23)\end{array}$ & $4.69(4.62-4.77)$ \\
\hline \multicolumn{5}{|l|}{ Wealth Status } \\
\hline Poor & $\begin{array}{l}34.39(34.17- \\
34.60)\end{array}$ & $6.49(6.38-6.61)$ & $\begin{array}{l}30.59(30.39- \\
30.80)\end{array}$ & $2.70(2.63-2.77)$ \\
\hline Middle & $\begin{array}{l}38.99(38.68- \\
39.29)\end{array}$ & 9.32 (9.14-9.51) & $\begin{array}{l}34.25(33.95- \\
34.55)\end{array}$ & $4.59(4.46-4.72)$ \\
\hline Rich & $\begin{array}{l}46.83(46.62- \\
47.05)\end{array}$ & $\begin{array}{l}17.40(17.23- \\
17.56)\end{array}$ & $\begin{array}{l}39.63(39.42- \\
39.85)\end{array}$ & $\begin{array}{l}10.20(10.07- \\
10.33)\end{array}$ \\
\hline \multicolumn{5}{|l|}{ Smoking Status } \\
\hline No & $\begin{array}{l}39.78(39.62- \\
39.93)\end{array}$ & $\begin{array}{l}11.59(11.49- \\
11.69)\end{array}$ & $\begin{array}{l}34.37(34.22- \\
34.52)\end{array}$ & $6.18(6.11-6.26)$ \\
\hline
\end{tabular}




\begin{tabular}{|c|c|c|c|c|}
\hline Independent & NCD & Diabetes & Hypertension & Comorbidity \\
\hline Variables & $\begin{array}{l}\text { Prevalence }(95 \% \\
\mathrm{Cl})\end{array}$ & $\begin{array}{l}\text { Prevalence } \\
(95 \% \mathrm{Cl})\end{array}$ & $\begin{array}{l}\text { Prevalence } \\
(95 \% \mathrm{Cl})\end{array}$ & $\begin{array}{l}\text { Prevalence } \\
(95 \% \mathrm{Cl})\end{array}$ \\
\hline Yes & $\begin{array}{l}43.33(43.00- \\
43.65)\end{array}$ & $\begin{array}{l}11.37(11.16- \\
11.58)\end{array}$ & $\begin{array}{l}38.03(37.72- \\
38.35)\end{array}$ & $6.07(5.92-6.23)$ \\
\hline \multicolumn{5}{|c|}{ Source of Drinking Water } \\
\hline Improved & $\begin{array}{l}40.51(40.37- \\
40.65)\end{array}$ & $\begin{array}{l}11.67(11.57- \\
11.76)\end{array}$ & $\begin{array}{l}35.06 \text { (34.93- } \\
35.20)\end{array}$ & $6.22(6.15-6.29)$ \\
\hline Unimproved & $\begin{array}{l}36.09(35.13- \\
37.05)\end{array}$ & $5.61(5.17-6.09)$ & $\begin{array}{l}33.88(32.94- \\
34.84)\end{array}$ & $3.41(3.07-3.79)$ \\
\hline \multicolumn{5}{|l|}{ Sanitation Facility } \\
\hline Improved & $\begin{array}{l}43.11(42.94- \\
43.28)\end{array}$ & $\begin{array}{l}13.07(12.96- \\
13.19)\end{array}$ & $\begin{array}{l}37.31(37.15- \\
37.48)\end{array}$ & $7.27(7.19,7.36)$ \\
\hline Unimproved & $\begin{array}{l}35.04 \text { (34.80- } \\
35.27)\end{array}$ & $8.54(8.41-8.68)$ & $\begin{array}{l}30.45(30.22- \\
30.68)\end{array}$ & $3.96(3.86,4.06)$ \\
\hline Open defecation 9 & $\begin{array}{l}35.82 \text { (34.66- } \\
36.99)\end{array}$ & $7.38(6.77-8.04)$ & $\begin{array}{l}31.97(30.85- \\
33.12)\end{array}$ & $3.54(3.11,4.01)$ \\
\hline \multicolumn{5}{|c|}{ 'Indicates no sanitation facility or open-air defecation. } \\
\hline \multicolumn{5}{|c|}{ NCD: Non-communicable disease; Cl: Confidence interval; BMI: Body mass index. } \\
\hline
\end{tabular}

\section{Factors associated with non-communicable diseases (NCDs)}

Chi-square analysis demonstrated that geographic location, place of residence, participants' age, BMI level, occupational status, wealth status and sanitary facility were significantly $(p<0.05)$ associated with NCD, diabetes, HT and co-morbidity (Table 3 ). 
Table 3

Chi-square and eta-square test of association for independent variables ( $N=12,093)$

\begin{tabular}{|c|c|c|c|c|c|c|c|c|}
\hline \multirow{2}{*}{$\begin{array}{l}\text { Independent } \\
\text { Variables }\end{array}$} & \multicolumn{2}{|c|}{$\operatorname{NCD}(n=3,986)$} & \multicolumn{2}{|c|}{ Diabetes $(n=1,201)$} & \multicolumn{2}{|c|}{$\begin{array}{l}\text { Hypertension } \\
(\mathrm{n}=3,338)\end{array}$} & \multicolumn{2}{|c|}{$\begin{array}{l}\text { Comorbidity } \\
(n=553)\end{array}$} \\
\hline & $\begin{array}{l}\text { Chi- } \\
\text { square } \\
\text { (p-value) }\end{array}$ & $\begin{array}{l}\text { Eta- } \\
\text { square }\end{array}$ & $\begin{array}{l}\text { Chi- } \\
\text { square } \\
\text { (p-value) }\end{array}$ & $\begin{array}{l}\text { Eta- } \\
\text { square }\end{array}$ & $\begin{array}{l}\text { Chi- } \\
\text { square } \\
\text { (p-value) }\end{array}$ & $\begin{array}{l}\text { Eta- } \\
\text { square }\end{array}$ & $\begin{array}{l}\text { Chi- } \\
\text { square } \\
\text { (p-value) }\end{array}$ & $\begin{array}{l}\text { Eta- } \\
\text { square }\end{array}$ \\
\hline $\begin{array}{l}\text { Geographic } \\
\text { Location }\end{array}$ & $\begin{array}{l}33.89 \\
(<0.0001)\end{array}$ & - & $\begin{array}{l}84.28 \\
(<0.0001)\end{array}$ & - & $\begin{array}{l}56.43 \\
(<0.0001)\end{array}$ & - & $\begin{array}{l}29.13 \\
(<0.0001)\end{array}$ & - \\
\hline $\begin{array}{l}\text { Place of } \\
\text { Residence }\end{array}$ & $\begin{array}{l}17.44 \\
(<0.0001)\end{array}$ & - & $\begin{array}{l}43.49 \\
(<0.0001)\end{array}$ & - & $\begin{array}{l}7.26 \\
(0.007)\end{array}$ & - & $\begin{array}{l}32.44 \\
(<0.0001)\end{array}$ & - \\
\hline $\begin{array}{l}\text { Participant's } \\
\text { Sex }\end{array}$ & $\begin{array}{l}2.60 \\
(0.1066)\end{array}$ & - & $\begin{array}{l}0.07 \\
(0.7843)\end{array}$ & - & $\begin{array}{l}6.64 \\
(0.009)\end{array}$ & - & $\begin{array}{l}2.27 \\
(0.1318)\end{array}$ & - \\
\hline $\begin{array}{l}\text { Participant's } \\
\text { Age }^{\circledR} \text { (Years) }\end{array}$ & $\begin{array}{l}1517.70 \\
(<0.0001)\end{array}$ & 0.37 & $\begin{array}{l}269.33 \\
(<0.0001)\end{array}$ & 0.17 & $\begin{array}{l}1521.70 \\
(<0.0001)\end{array}$ & 0.37 & $\begin{array}{l}353.26 \\
(<0.0001)\end{array}$ & 0.19 \\
\hline BMI Level $^{\rrbracket}$ & $\begin{array}{l}492.35 \\
(<0.0001)\end{array}$ & 0.23 & $\begin{array}{l}189.74 \\
(<0.0001)\end{array}$ & 0.14 & $\begin{array}{l}457.25 \\
(<0.0001)\end{array}$ & 0.21 & $\begin{array}{l}240.66 \\
(<0.0001)\end{array}$ & 0.16 \\
\hline $\begin{array}{l}\text { Education } \\
\text { Level }\end{array}$ & $\begin{array}{l}133.08 \\
(<0.0001)\end{array}$ & - & $\begin{array}{l}0.56 \\
(0.9062)\end{array}$ & - & $\begin{array}{l}165.36 \\
(<0.0001)\end{array}$ & - & $\begin{array}{l}3.18 \\
(0.3654)\end{array}$ & - \\
\hline $\begin{array}{l}\text { Occupational } \\
\text { Status }\end{array}$ & $\begin{array}{l}72.48 \\
(<0.0001)\end{array}$ & - & $\begin{array}{l}31.89 \\
(<0.0001)\end{array}$ & - & $\begin{array}{l}63.79 \\
(<0.0001)\end{array}$ & - & $\begin{array}{l}35.56 \\
(<0.0001)\end{array}$ & - \\
\hline $\begin{array}{l}\text { Wealth } \\
\text { Status }\end{array}$ & $\begin{array}{l}134.22 \\
(<0.0001)\end{array}$ & - & $\begin{array}{l}219.44 \\
(<0.0001)\end{array}$ & - & $\begin{array}{l}75.88 \\
(<0.0001)\end{array}$ & - & $\begin{array}{l}181.23 \\
(<0.0001)\end{array}$ & - \\
\hline $\begin{array}{l}\text { Smoking } \\
\text { Status }\end{array}$ & $\begin{array}{l}22.74 \\
(<0.0001)\end{array}$ & - & $\begin{array}{l}1.61 \\
(0.205)\end{array}$ & - & $\begin{array}{l}21.81 \\
(<0.0001)\end{array}$ & - & $\begin{array}{l}1.20 \\
(0.2734)\end{array}$ & - \\
\hline $\begin{array}{l}\text { Source of } \\
\text { Drinking } \\
\text { Water }\end{array}$ & $\begin{array}{l}1.936 \\
(0.1641)\end{array}$ & - & $\begin{array}{l}5.56 \\
(0.1836)\end{array}$ & - & $\begin{array}{l}0.37 \\
(0.5421)\end{array}$ & - & $\begin{array}{l}2.28 \\
(0.1311)\end{array}$ & - \\
\hline $\begin{array}{l}\text { Sanitation } \\
\text { Facility }\end{array}$ & $\begin{array}{l}62.74 \\
(<0.0001)\end{array}$ & - & $\begin{array}{l}43.51 \\
(<0.0001)\end{array}$ & - & $\begin{array}{l}46.95 \\
(<0.0001)\end{array}$ & - & $\begin{array}{l}37.69 \\
(<0.0001)\end{array}$ & - \\
\hline \multicolumn{9}{|c|}{$\begin{array}{l}{ }^{\mathbb{B}} \text { Both chi-square and eta-square test statistic were calculated for participant's age and BMI level. In chi-square } \\
\text { test, participant's age }(18-24,25-34,35-44,45-54,55-64,>=65) \text { and BMI level (underweight: }<18.5 \mathrm{~kg} / \mathrm{m}^{2}, \\
\left.\text { normal: } 18.5-24.9 \mathrm{~kg} / \mathrm{m}^{2}, \text { overweight: } 25.0-29.9 \mathrm{~kg} / \mathrm{m}^{2} \text {, and obesity: } \geq 30.0 \mathrm{~kg} / \mathrm{m}^{2}\right) \text { were categorical. Whereas, } \\
\text { both variables were in continuous scale for eta-square test of association. NCD: Non-communicable disease; } \\
p \text {-value: Probability value; BMl: Body mass index. }\end{array}$} \\
\hline
\end{tabular}

The adjusted estimated effect of factors associated with NCD are shown in Table 4. Respondents from Barishal [adjusted Prevalence Ratio, $(\mathrm{APR})=1.10 ; 95 \% \mathrm{Cl}$ : 1.02-1.18] and Rangpur division (APR $=1.07 ; 95 \% \mathrm{Cl}$ : 1.00-1.14) were more likely to be associated with NCD compared to those from Dhaka division. Respondents whose age above 34 years old were more likely to be associated with NCD, DM, HT, and co-morbidity with the increment of ages as compared to their counterparts. NCD, DM, HT and co-morbidity were found to be lower among underweight participants and higher among overweight participants than in normal weight individuals. Participants who involved in work as an occupation were low risk for suffering from CND (APR $=0.93 ; 95 \% \mathrm{Cl}$ : 
0.89-0.96), DM (APR = 0.85 95\% Cl: 0.76-0.95) and co-morbidity (APR $=0.80 ; 95 \%$ Cl: 0.68-0.95) compared to their counterparts. Respondents from rich wealth status were at higher-risk of NCD (APR = 1.09; 95\% Cl: 1.04-1.15), DM $(A P R=1.97 ; 95 \% \mathrm{Cl}: 1.70-2.29)$ and comorbidity (APR $=2.43 ; 95 \% \mathrm{Cl}: 1.92-3.10)$ compared to those who from poor wealth condition. Similarly, smoker participants had higher prevalence of NCD (APR $=1.94 ; 95 \%$ Cl: $1.89-1.98)$ and $\mathrm{HT}(\mathrm{APR}=1.91 ; 95 \% \mathrm{Cl}: 1.86-1.97)$ than non-smoker participants. Females were more tend to be hypertensive $(A P R=1.10 ; 95 \% \mathrm{Cl}: 1.05-1.16)$ compared to their male counterparts (Table 4). 
Table 4

Estimation of adjusted prevalence ratio for risk factors associated with non-communicable disease $(\mathrm{N}=12,093)$

\begin{tabular}{|c|c|c|c|c|c|c|c|c|}
\hline \multirow[t]{2}{*}{ Variables } & \multicolumn{2}{|c|}{$\operatorname{NCD}(n=3,986)$} & \multicolumn{2}{|c|}{ Diabetes $(n=1,201)$} & \multicolumn{2}{|c|}{$\begin{array}{l}\text { Hypertension } \\
(n=3,338)\end{array}$} & \multicolumn{2}{|c|}{$\begin{array}{l}\text { Co-morbidity } \\
(n=553)\end{array}$} \\
\hline & $\begin{array}{l}\text { APR } \\
\text { (95\% } \\
\text { Cl) }\end{array}$ & p-value & $\begin{array}{l}\text { APR } \\
\text { (95\% } \\
\text { Cl) }\end{array}$ & p-value & $\begin{array}{l}\text { APR } \\
\text { (95\% } \\
\text { Cl) }\end{array}$ & p-value & $\begin{array}{l}\text { APR } \\
\text { (95\% } \\
\text { Cl) }\end{array}$ & p-value \\
\hline
\end{tabular}

\section{Geographic Location}

\begin{tabular}{|c|c|c|c|c|c|c|c|c|}
\hline Dhaka (Ref.) & 1 & - & 1 & - & 1 & - & 1 & - \\
\hline Barisal & $\begin{array}{l}1.10 \\
(1.02- \\
1.18)\end{array}$ & 0.0119 & $\begin{array}{l}0.80 \\
(0.62- \\
1.02)\end{array}$ & 0.0764 & $\begin{array}{l}1.19 \\
(1.09- \\
1.29)\end{array}$ & $<0.0001$ & $\begin{array}{l}0.87 \\
(0.58- \\
1.26)\end{array}$ & 0.4966 \\
\hline Chittagong & $\begin{array}{l}1.05 \\
(0.99- \\
1.11)\end{array}$ & 0.0862 & $\begin{array}{l}0.82 \\
(0.71- \\
0.95)\end{array}$ & 0.0083 & $\begin{array}{l}1.13 \\
(1.06- \\
1.21)\end{array}$ & 0.0003 & $\begin{array}{l}1.03 \\
(0.83- \\
1.29)\end{array}$ & 0.7686 \\
\hline Khulna & $\begin{array}{l}1.00 \\
(0.93- \\
1.06)\end{array}$ & 0.8758 & $\begin{array}{l}0.60 \\
(0.49- \\
0.72)\end{array}$ & $<0.0001$ & $\begin{array}{l}1.10 \\
(1.02- \\
1.18)\end{array}$ & 0.0177 & $\begin{array}{l}0.82 \\
(0.62- \\
1.07)\end{array}$ & 0.1499 \\
\hline Mymensingh & $\begin{array}{l}0.96 \\
(0.89- \\
1.05)\end{array}$ & 0.3684 & $\begin{array}{l}0.71 \\
(0.56- \\
0.89)\end{array}$ & 0.0031 & $\begin{array}{l}1.02 \\
(0.92- \\
1.12)\end{array}$ & 0.8007 & $\begin{array}{l}0.78 \\
(0.53- \\
1.11)\end{array}$ & 0.1961 \\
\hline Rajshahi & $\begin{array}{l}1.03 \\
(0.97- \\
1.10)\end{array}$ & 0.3497 & $\begin{array}{l}0.69 \\
(0.57- \\
0.82)\end{array}$ & $<0.0001$ & $\begin{array}{l}1.11 \\
(1.03- \\
1.19)\end{array}$ & 0.0068 & $\begin{array}{l}0.76 \\
(0.56- \\
1.01)\end{array}$ & 0.0633 \\
\hline Rangpur & $\begin{array}{l}1.07 \\
(1.00- \\
1.14)\end{array}$ & 0.0360 & $\begin{array}{l}0.53 \\
(0.42- \\
0.67)\end{array}$ & $<0.0001$ & $\begin{array}{l}1.20 \\
(1.11- \\
1.30)\end{array}$ & $<0.0001$ & $\begin{array}{l}0.70 \\
(0.49- \\
0.97)\end{array}$ & 0.0370 \\
\hline Sylhet & $\begin{array}{l}1.04 \\
(0.96- \\
1.13)\end{array}$ & 0.3732 & $\begin{array}{l}0.79 \\
(0.62- \\
0.99)\end{array}$ & 0.0441 & $\begin{array}{l}1.12 \\
(1.01- \\
1.23)\end{array}$ & 0.0254 & $\begin{array}{l}0.94 \\
(0.64- \\
1.33)\end{array}$ & 0.7361 \\
\hline
\end{tabular}

Place of Residence

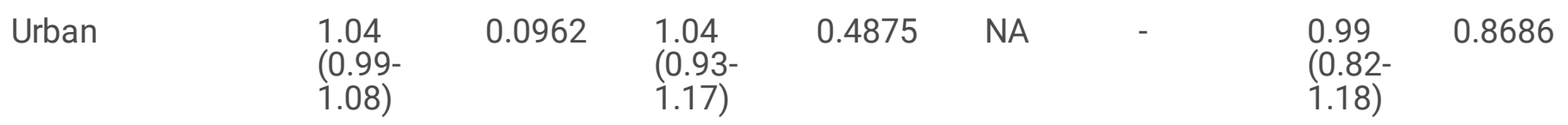

Rural (Ref.)

1

1

NA

1

\section{Participant's Sex}

\begin{tabular}{|c|c|c|c|c|c|c|c|}
\hline Male (Ref.) & NA & - & NA & - & 1 & - & NA \\
\hline Female & NA & & NA & - & $\begin{array}{l}1.10 \\
(1.05- \\
1.16)\end{array}$ & 0.0003 & NA \\
\hline
\end{tabular}

\section{Participant's Age \\ (Years)}




\begin{tabular}{|c|c|c|c|c|c|c|c|c|}
\hline \multirow[t]{2}{*}{ Variables } & \multicolumn{2}{|c|}{$\operatorname{NCD}(n=3,986)$} & \multicolumn{2}{|c|}{ Diabetes $(n=1,201)$} & \multicolumn{2}{|c|}{$\begin{array}{l}\text { Hypertension } \\
(n=3,338)\end{array}$} & \multicolumn{2}{|c|}{$\begin{array}{l}\text { Co-morbidity } \\
(n=553)\end{array}$} \\
\hline & $\begin{array}{l}\text { APR } \\
(95 \% \\
\text { Cl) }\end{array}$ & p-value & $\begin{array}{l}\text { APR } \\
(95 \% \\
\text { Cl) }\end{array}$ & p-value & $\begin{array}{l}\text { APR } \\
(95 \% \\
\text { Cl) }\end{array}$ & p-value & $\begin{array}{l}\text { APR } \\
(95 \% \\
\text { Cl) }\end{array}$ & p-value \\
\hline $35-44$ & $\begin{array}{l}1.50 \\
(1.41- \\
1.59)\end{array}$ & $<0.0001$ & $\begin{array}{l}2.17 \\
(1.85- \\
2.54)\end{array}$ & $<0.0001$ & $\begin{array}{l}1.60 \\
(1.49- \\
1.72)\end{array}$ & $<0.0001$ & $\begin{array}{l}3.93 \\
(2.89- \\
5.39)\end{array}$ & $<0.0001$ \\
\hline $45-54$ & $\begin{array}{l}1.82 \\
(1.71- \\
1.93)\end{array}$ & $<0.0001$ & $\begin{array}{l}2.94 \\
(2.50- \\
3.44)\end{array}$ & $<0.0001$ & $\begin{array}{l}2.03 \\
(1.88- \\
2.18)\end{array}$ & $<0.0001$ & $\begin{array}{l}7.20 \\
(5.37- \\
9.77)\end{array}$ & $<0.0001$ \\
\hline $55-64$ & $\begin{array}{l}2.10 \\
(1.98- \\
2.23)\end{array}$ & $<0.0001$ & $\begin{array}{l}3.25 \\
(2.75- \\
3.83)\end{array}$ & $<0.0001$ & $\begin{array}{l}2.46 \\
(2.28- \\
2.64)\end{array}$ & $<0.0001$ & $\begin{array}{l}9.22 \\
(6.89- \\
12.50)\end{array}$ & $<0.0001$ \\
\hline$>=65$ & $\begin{array}{l}2.28 \\
(2.15- \\
2.42)\end{array}$ & $<0.0001$ & $\begin{array}{l}3.46 \\
(2.65- \\
3.78)\end{array}$ & $<0.0001$ & $\begin{array}{l}2.80 \\
(2.60- \\
3.02)\end{array}$ & $<0.0001$ & $\begin{array}{l}9.76 \\
(7.21- \\
13.35)\end{array}$ & $<0.0001$ \\
\hline \multicolumn{9}{|l|}{ BMI Level } \\
\hline $\begin{array}{l}\text { Underweight } \\
\left(<18.5 \mathrm{~kg} / \mathrm{m}^{2}\right)\end{array}$ & $\begin{array}{l}0.82 \\
(0.77- \\
0.87)\end{array}$ & $<0.0001$ & $\begin{array}{l}0.75 \\
(0.63- \\
0.91)\end{array}$ & 0.0025 & $\begin{array}{l}0.77 \\
(0.72- \\
0.83)\end{array}$ & $<0.0001$ & $\begin{array}{l}0.44 \\
(0.30- \\
0.63)\end{array}$ & $<0.0001$ \\
\hline $\begin{array}{l}\text { Normal }(18.5- \\
\left.24.9 \mathrm{~kg} / \mathrm{m}^{2}\right) \\
\text { (Ref.) }\end{array}$ & 1 & - & 1 & - & 1 & - & 1 & - \\
\hline $\begin{array}{l}\text { Overweight (25.0- } \\
\left.29.9 \mathrm{~kg} / \mathrm{m}^{2}\right)\end{array}$ & $\begin{array}{l}1.33 \\
(1.27- \\
1.39)\end{array}$ & $<0.0001$ & $\begin{array}{l}1.40 \\
\text { (1.24- } \\
1.58)\end{array}$ & $<0.0001$ & $\begin{array}{l}1.41 \\
\text { (1.34- } \\
1.48)\end{array}$ & $<0.0001$ & $\begin{array}{l}1.96 \\
(1.63- \\
2.35)\end{array}$ & $<0.0001$ \\
\hline $\begin{array}{l}\text { Obesity }(\geq 30.0 \\
\left.\mathrm{kg} / \mathrm{m}^{2}\right)\end{array}$ & $\begin{array}{l}1.35 \\
(1.26- \\
1.44)\end{array}$ & $<0.0001$ & $\begin{array}{l}1.63 \\
(1.34- \\
1.97)\end{array}$ & $<0.0001$ & $\begin{array}{l}1.43 \\
(1.33- \\
1.55)\end{array}$ & $<0.0001$ & $\begin{array}{l}2.51 \\
(1.92- \\
3.22)\end{array}$ & $<0.0001$ \\
\hline \multicolumn{9}{|l|}{ Education Level } \\
\hline $\begin{array}{l}\text { No Education } \\
\text { (Ref.) }\end{array}$ & 1 & - & NA & - & 1 & - & NA & 1 \\
\hline Primary & $\begin{array}{l}1.02 \\
(0.97- \\
1.07)\end{array}$ & 0.4557 & NA & - & $\begin{array}{l}0.99 \\
(0.94- \\
1.05)\end{array}$ & 0.8661 & NA & - \\
\hline Secondary & $\begin{array}{l}1.03 \\
(0.97- \\
1.08)\end{array}$ & 0.3415 & NA & - & $\begin{array}{l}1.03 \\
(0.97- \\
1.10)\end{array}$ & 0.3461 & NA & - \\
\hline Higher & $\begin{array}{l}1.00 \\
(0.94- \\
1.08)\end{array}$ & 0.8151 & NA & - & $\begin{array}{l}1.01 \\
(0.94- \\
1.11)\end{array}$ & 0.6522 & NA & - \\
\hline \multicolumn{9}{|l|}{ Occupational Status } \\
\hline $\begin{array}{l}\text { Not Working } \\
\text { (Ref.) }\end{array}$ & 1 & - & 1 & - & 1 & - & 1 & - \\
\hline
\end{tabular}




\begin{tabular}{|c|c|c|c|c|c|c|c|c|}
\hline \multirow[t]{2}{*}{ Variables } & \multicolumn{2}{|c|}{$\operatorname{NCD}(n=3,986)$} & \multicolumn{2}{|c|}{ Diabetes $(n=1,201)$} & \multicolumn{2}{|c|}{$\begin{array}{l}\text { Hypertension } \\
(n=3,338)\end{array}$} & \multicolumn{2}{|c|}{$\begin{array}{l}\text { Co-morbidity } \\
(n=553)\end{array}$} \\
\hline & $\begin{array}{l}\text { APR } \\
(95 \% \\
\mathrm{Cl})\end{array}$ & p-value & $\begin{array}{l}\text { APR } \\
(95 \% \\
\mathrm{Cl})\end{array}$ & p-value & $\begin{array}{l}\text { APR } \\
(95 \% \\
\mathrm{Cl})\end{array}$ & p-value & $\begin{array}{l}\text { APR } \\
(95 \% \\
\mathrm{Cl})\end{array}$ & p-value \\
\hline Working & $\begin{array}{l}0.93 \\
(0.89- \\
0.96)\end{array}$ & 0.0002 & $\begin{array}{l}0.85 \\
(0.76- \\
0.95)\end{array}$ & 0.0034 & $\begin{array}{l}0.96 \\
(0.91- \\
1.01)\end{array}$ & 0.1181 & $\begin{array}{l}0.80 \\
(0.68- \\
0.95)\end{array}$ & 0.0105 \\
\hline \multicolumn{9}{|l|}{ Wealth Status } \\
\hline Poor (Ref.) & 1 & - & 1 & - & 1 & - & 1 & - \\
\hline Middle & $\begin{array}{l}1.03 \\
(0.97- \\
1.09)\end{array}$ & 0.3430 & $\begin{array}{l}1.24 \\
(1.04- \\
1.48)\end{array}$ & 0.0192 & $\begin{array}{l}1.02 \\
(0.96- \\
1.09)\end{array}$ & 0.4873 & $\begin{array}{l}1.49 \\
(1.12- \\
1.97)\end{array}$ & 0.0063 \\
\hline Rich & $\begin{array}{l}1.09 \\
(1.04- \\
1.15)\end{array}$ & 0.0007 & $\begin{array}{l}1.97 \\
(1.70- \\
2.29)\end{array}$ & $<0.0001$ & $\begin{array}{l}1.05 \\
(1.00- \\
1.12)\end{array}$ & 0.0528 & $\begin{array}{l}2.43 \\
(1.92- \\
3.10)\end{array}$ & $<0.0001$ \\
\hline \multicolumn{9}{|l|}{ Smoking Status } \\
\hline No (Ref.) & 1 & - & NA & - & 1 & - & NA & - \\
\hline Yes & $\begin{array}{l}1.94 \\
(1.89- \\
1.98)\end{array}$ & 0.0088 & NA & & $\begin{array}{l}1.91 \\
(1.86- \\
1.97)\end{array}$ & 0.0022 & NA & - \\
\hline \multicolumn{9}{|c|}{$\begin{array}{l}\text { NA stands for not applicable. Insignificant variables (i.e., } p \text {-value }<0.05 \text { ) in unadjusted regression models were } \\
\text { not included in the adjusted models; therefore, they were replaced with NA in the Table. NCD: Non- } \\
\text { communicable disease; APR: adjusted Prevalence Ratio; Cl: Confidence Interval; } p \text {-value: Probability value; Ref.: } \\
\text { Reference category; BMl: Body mass index. }\end{array}$} \\
\hline
\end{tabular}

\section{Discussion}

NCDs have become a major public health challenge in Bangladesh with a higher prevalence of double burden [29]. This study focused on the estimation of age-standardized prevalence and factors associated with the of DM, HT and comorbidity (both DM and HT) among adult population in Bangladesh. Similar to the previous studies [15, 29-33], we found that females, aged over 35 years and higher, having overweight or obesity, being from households with rich wealth status were the significant risk factors associated with any of the NCDs studied. While participants' current working status were associated with the lower odds of having NCDs. The findings of this study revealed that the overall age-adjusted prevalence of DM and HT was $11.55 \%$ and $35.04 \%$, respectively, and $6.16 \%$ had comorbidity which are higher than the prevalence of DM (11.0\%), HT (29.7\%), and comorbidity (4.5\%) among Bangladeshi adult population found in 2011[29]. Though the DM prevalence is almost consistent, the prevalence of HT was higher than the overall prevalence (age-adjusted) of South-Asian countries $(20.1 \%)$ and other low-middle-income countries (31.5\%)[34]. These increasing pattern and higher prevalence of NCDs indicate that Bangladesh is in a great challenge in controlling as well as to reduce the burden of chronic diseases. This rising burden could be due to the epidemiological transition of Bangladesh like life expectancy at birth [32], increasing number of ageing population, rapid urbanization, lifestyle changes and physical inactivity [34]. In order to curve the burden of these NCDs, the government of Bangladesh should focus on implementing the awareness, 
prevention and control programs, since such type of programs in Bangladesh are insufficient reported by previous study [35].

This study observed that females were more prone to be hypertensive compared to their male counterparts. This finding aligns with other similar studies conducted in Bangladesh [29-33]. Various biological and environmental factors could be responsible for this higher prevalence among females [36]. In addition, several studies have shown that stress is often linked to high blood pressure, and middle-aged women may be more susceptible to have higher stress especially during menopause [37,38]. Again, prevalence of overweight and obesity were found to be higher among females than males previously [39], and an increased BMI might also be associated with the elevated blood pressure [29]. Previous literatures have also shown that differences in physiological and behavioral characteristics between males and females may contribute to these differences [40, 41].

We found a positive relationship between age and prevalence of NCDs where the risk of NCDs were increased with the increase of age. Age is sometimes considered as an irreversible risk factors of different NCDs [42-44].

Presently, Bangladesh is undergoing a demographic change which may result in a large proportion of the elderly in this country [12]. Evidence shows that older people are at greater risk of having different chronic conditions like diabetes, hypertension, overweight/obesity, etc.,[24, 42, 44, 45]. These diseases affect each other's outcomes, and also they have many common risk factors that can lead to more serious complications [24, 42]. The higher tendency of these NCDs among older adults could be due to various health problems happen among older people and their lifestyle like poor nutritional condition, stiffness, sodium intake, physical inactivity, and lower immunity $[42,43,46]$.

Participants with BMI higher than normal were at higher risk of suffering chronic diseases; such positive link between higher BMI and NCDs are well-established and can be justified by the previous evidence $[15,30,32,33]$. Bangladesh is undergoing a greater challenge in controlling the NCDs while the prevalence of overweight and obesity is uprising $[47,48]$. Along with the nutritional changes in Bangladesh, the steady rise in BMI has serious consequences related to premature NCD-related illness and death. Various metabolic and genetic factors might be responsible for such positive association [49-51]. Thus, controlling and preventing the NCDs along with overweight/obesity would be fruitful since prevention and controlling measures of overweight/obesity and other chronic conditions are almost similar [15]. Thus, simultaneously controlling these conditions will have a significant positive impact on the health system in Bangladesh [52, 53].

As found in the previous literatures $[29,54]$, present study found that people involved in any type of work were at reduced risk of diabetes and comorbidity. Involving in work increases the physical activity and several studies found a negative association between moderate/higher levels of physical activities and chronic diseases $[55,56]$. Physical activity increases the use of oxygen throughout the body and maintains blood glucose levels [57]. In addition, physical activity also reduces the adverse consequences of many chronic diseases like diabetes [58]; hence, promoting physical activity is crucial in controlling diabetes and other chronic diseases $[59,60]$.

Compatible with earlier studies $[15,29]$, we observed that individual with higher economic status were at increased risk of having DM and comorbidity (coexistence of both DM and HT) compared to those belong to poor households. Another Bangladeshi study identified that the odds of having DM and comorbidity were higher among people from rich families [61] which is similar to what we found in this study. A plausible reason for this finding might be due to some factors including lower physical activity, sedentary lifestyle, and higher overweight/obesity among people from richest quintile $[29,61]$. Besides, people with relatively low socio-economic status in

Page 17/23 
Bangladesh are employed in more laborious work that may keep them away from sedentary lifestyle and they are also supposed to eat foods with less amount of calories [62, 63].

Similar to previous studies $[64,65]$, we also found that smoking is positively associated with NCD and HT. It is well-established by previous several global studies $[64,65]$ that unhealthy lifestyle like smoking is associated with increased risk of chronic diseases. Though available evidence from prospective studies already identified smoking as a confirmed risk factor for hypertension[66] and other NCDs [67], the relationship between chronic smoking and raised blood pressure is still controversial [68]. Furthermore, smoking can have a greater detrimental effect on central blood pressure by affecting arterial stiffness and wave reflection that may explain the causal relationship between smoking and developing hypertension[69].

This study's strengths and limitations have been acknowledged. The generalizability of the findings for Bangladesh is a strength of this study, as this survey included nationally representative data from all divisions. In addition, appropriate statistical procedures were used to assess the sample's weighted prevalence of hypertension. The study's limitations are stated in a fair manner. No causality could be established due to the cross-sectional design, and the participants' blood pressure was taken three times in one day.

\section{Conclusions}

Adults in Bangladesh suffer from an unprecedentedly high prevalence of hypertension, diabetes, and comorbidity, pushing policymakers and public health practitioners to design population-specific drug and lifestyle treatment strategies. Thus, the findings of this study suggest that immediate policy measures should be made to target this age group for the prevention, early detection, and treatment of NCDs. The evidence obtained in this study may be extremely useful in developing a community-based study to better understand modifiable factors (such as food habits, physical activity, salt intake, and stress).

\section{Abbreviations}

NCD: Non-communicable disease; DM: Diabetes Mellitus; HT: Hypertension; BDHS: Bangladesh Demographic and Health Survey; PSU: Primary Sampling Unit; BMI: Body Mass Index.

\section{Declarations}

\section{Ethics approval and consent to participate}

\section{Ethical consideration}

We did not need any further ethical permission because we used secondary data from the Demographic and Health Surveys (DHS) Program, which is publicly available. In the BDHS report, the DHS Program's ethical procedures are described in depth[12]. All the procedures were performed in accordance with the relevant guidelines and regulations.

\section{Consent for publication}

Not applicable

Availability of data and materials 
This study used publicly available Demographic and Health Surveys Program datasets from Bangladesh which can be freely obtained from https://dhsprogram.com/. As a third-party user, we don't have permission to share the data publicly in any platforms.

\section{Competing interests}

None of the authors declares any conflict of interest.

\section{Funding}

This research did not receive any specific grant from any financial and non-financial sectors.

\section{Author Contributions:}

MAR, HRH conceptualized the study design. MAR and $\mathrm{HRH}$, had all access to the data and validation of the statistical analysis and did the formal analysis and drafting the original manuscript. MAR, SK and HB helped in drafting the original manuscript. MAR, $\mathrm{HRH}, \mathrm{SK}$ and writing and critically reviewed the manuscript. MAR supervised the whole study.

\section{Acknowledgement}

The authors declare no conflict of interest. The authors would like to thank the Demographic and health survey for providing the data freely.

\section{Data availability}

The dataset of BDHS 2017-18 is available upon request in the following website: http://dhsprogram.com/data/available-datasets.cfm.

\section{References}

1. World Health Organization. Noncommunicable diseases: Progress monitor 2020. 2020.

2. Khan JAM, Trujillo AJ, Ahmed S, Siddiquee AT, Alam N, Mirelman AJ, et al. Distribution of chronic disease mortality and deterioration in household socioeconomic status in rural Bangladesh: an analysis over a 24year period. Int J Epidemiol. 2015;44:1917-26.

3. Khalequzzaman M, Chiang C, Choudhury SR, Yatsuya H, Al-Mamun MA, Al-Shoaibi AAA, et al. Prevalence of non-communicable disease risk factors among poor shantytown residents in Dhaka, Bangladesh: $A$ community-based cross-sectional survey. BMJ Open. 2017;7.

4. Bennett JE, Stevens GA, Mathers CD, Bonita R, Rehm J, Kruk ME, et al. NCD Countdown 2030: worldwide trends in non-communicable disease mortality and progress towards Sustainable Development Goal target 3.4. Lancet. 2018;392:1072-88.

5. World Health Organization. WHO | Global Action Plan for the Prevention and Control of NCDs 2013-2020. Geneva, Switzerland; 2013. https://www.who.int/nmh/events/ncd_action_plan/en/. Accessed 14 Apr 2020.

6. Ahsan KZ, Alam MN, Streatfield PK, Nahar Q. Has Bangladesh Entered the Fourth Stage of the Epidemiologic Transition. In: Proceedings of the International seminar on Mortality: Past, Present and Future, the University of Campinas, Brazil. 2017. 
7. Al-Zubayer MA, Ahammed B, Sarder MA, Kundu S, Majumder UK, Islam SMS. Double and triple burden of noncommunicable diseases and its determinants among adults in Bangladesh: Evidence from a recent demographic and health survey. Int J Clin Pract. 2021; May:1-13.

8. Islam SMS, Purnat TD, Phuong NTA, Mwingira U, Schacht K, Fröschl G. Non-Communicable Diseases (NCDs) in developing countries: a symposium report. Global Health. 2014;10:1-8.

9. Nujhat S, Alam W, Parajuli A, Mohsen WAM, Banyira L, Gupta R Das, et al. Prevalence of risk factors for noncommunicable diseases in a rural population of Bangladesh: a cross-sectional study. Lancet Glob Heal. 2020;8:S21. doi:10.1016/s2214-109x(20)30162-5.

10. Riaz BK, Islam MZ, Islam ANMS, Zaman MM, Hossain MA, Rahman MM, et al. Risk factors for noncommunicable diseases in Bangladesh: Findings of the population-based cross-sectional national survey 2018. BMJ Open. 2020;10:1-11.

11. Bista $B$, Dhimal M, Bhattarai $S$, Neupane $T, X u$ YY, Pandey AR, et al. Prevalence of non-communicable diseases risk factors and their determinants: Results from STEPS survey 2019, Nepal. PLoS One. 2021;16 7 July:1-20. doi:10.1371/journal.pone.0253605.

12. National Institute of Population Research and Training (NIPORT), ICF International. Bangladesh Demographic and Health Survey 2017-18. Dhaka, Bangladesh, and Rockville, Maryland, USA; 2020. https://dhsprogram.com/pubs/pdf/PR104/PR104.pdf.

13. Rahman MA, Halder HR, Yadav UN, Mistry SK. Prevalence of and factors associated with hypertension according to JNC 7 and ACC/AHA 2017 guidelines in Bangladesh. Sci Rep. 2021;11:1-10.

14. Islam R, Khan MN, Oldroyd JC, Rana J, Chowdhury EK, Karim MN, et al. Prevalence of diabetes and prediabetes among Bangladeshi adults and associated factors: Evidence from the Demographic and Health Survey, 2017-18. medRxiv. 2021.

15. Al Kibria GM. Prevalence and Factors Associated with Diabetes among Bangladeshi Adults: An Analysis of Demographic and Health Survey 2017-18. Diabetes Epidemiol Manag. 2021;2:100012.

16. Forouzanfar MH, Afshin A, Alexander LT, Biryukov S, Brauer M, Cercy K, et al. Global, regional, and national comparative risk assessment of 79 behavioural, environmental and occupational, and metabolic risks or clusters of risks, 1990-2015: a systematic analysis for the Global Burden of Disease Study 2015. Lancet. 2016;388:1659-724.

17. Bista B, Dhungana RR, Chalise B, Pandey AR. Prevalence and determinants of noncommunicable diseases risk factors among reproductive aged women of Nepal: Results from Nepal demographic Health Survey 2016. PLoS One. 2020;15:1-13. doi:10.1371/journal.pone.0218840.

18. Rawal LB, Kanda K, Mahumud RA, Joshi D, Mehata S, Shrestha N, et al. Prevalence of underweight, overweight and obesity and their associated risk factors in Nepalese adults: Data from a nationwide survey, 2016. PLoS One. 2018;13:1-14.

19. World Health Organization. Noncommunicable diseases. https://www.who.int/news-room/factsheets/detail/noncommunicable-diseases. Accessed 11 Apr 2020.

20. Organization WH. Definition and diagnosis of diabetes mellitus and intermediate hyperglycaemia: report of a WHO/IDF consultation. 2006.

21. Whelton PK, Carey RM, Aronow WS. Acc/aha/aapa/abc/acpm/ags/APhA/ASH/ASPC/nma/pcna guideline for the prevention, Detection, evaluation, and management of high blood pressure in adults: a Report of the American College of Cardiology/American heart Association. Task force on clinical practi. Почки. 2018;7. 
22. Kibria GM Al, Swasey K, Kc A, Mirbolouk M, Sakib MN, Sharmeen A, et al. Estimated Change in Prevalence of Hypertension in Nepal Following Application of the 2017 ACC/AHA Guideline. JAMA Netw open. 2018;1:e180606.

23. Kibria GM Al, Swasey K, Choudhury A, Burrowes V, Stafford KA, Uddin SMI, et al. The new 2017 ACC/AHA guideline for classification of hypertension: changes in prevalence of hypertension among adults in Bangladesh. J Hum Hypertens. 2018;32:608-16.

24. Al Kibria GM, Swasey K, Hasan MZ, Choudhury A, Gupta R Das, Abariga SA, et al. Determinants of hypertension among adults in Bangladesh as per the Joint National Committee 7 and 2017 American College of Cardiology/American Hypertension Association hypertension guidelines. J Am Soc Hypertens. 2018;12:e45-55.

25. Kibria GM Al, Swasey K, Das Gupta R, Choudhury A, Nayeem J, Sharmeen A, et al. Differences in prevalence and determinants of hypertension according to rural-urban place of residence among adults in Bangladesh. $J$ Biosoc Sci. 2019;51:578-90.

26. Das Gupta R, Bin Zaman S, Wagle K, Crispen R, Hashan MR, Al Kibria GM. Factors associated with hypertension among adults in Nepal as per the Joint National Committee 7 and 2017 American College of Cardiology/American Heart Association hypertension guidelines: A cross-sectional analysis of the demographic and health survey 20. BMJ Open. 2019;9:1-8.

27. Chowdhury MAB, Uddin MJ, Haque MR, Ibrahimou B. Hypertension among adults in Bangladesh: evidence from a national cross-sectional survey. BMC Cardiovasc Disord. 2016;16:22.

28. World Health Organization. BMI Classification 2019. 2019. http://apps.who.int/bmi/index.jsp? introPage=intro_3.html. Accessed 18 Oct 2019.

29. Ali N, Akram R, Sheikh N, Sarker AR, Sultana M. Sex-specific prevalence, inequality and associated predictors of hypertension, diabetes, and comorbidity among Bangladeshi adults: results from a nationwide crosssectional demographic and health survey. BMJ Open. 2019;9:e029364.

30. Kibria A, Muhammed G, Gupta R Das, Nayeem J. Prevalence, awareness, and control of hypertension among Bangladeshi adults: an analysis of demographic and health survey 2017-18. Clin Hypertens. 2021;27:1-11.

31. Rahman MM, Gilmour S, Akter S, Abe SK, Saito E, Shibuya K. Prevalence and control of hypertension in Bangladesh: a multilevel analysis of a nationwide population-based survey. J Hypertens. 2015;33:465-72.

32. Khan MN, Oldroyd JC, Chowdhury EK, Hossain MB, Rana J, Renzetti S, et al. Prevalence, awareness, treatment, and control of hypertension in Bangladesh: Findings from National Demographic and Health Survey, 20172018. J Clin Hypertens (Greenwich).

33. Iqbal A, Ahsan KZ, Jamil K, Haider MM, Khan SH, Chakraborty N, et al. Demographic, socioeconomic, and biological correlates of hypertension in an adult population: evidence from the Bangladesh demographic and health survey 2017-18. BMC Public Health. 2021;21:1-14.

34. Mills KT, Bundy JD, Kelly TN, Reed JE, Kearney PM, Reynolds K, et al. Global disparities of hypertension prevalence and control: a systematic analysis of population-based studies from 90 countries. Circulation. 2016;134:441-50.

35. Biswas T, Pervin S, Tanim MIA, Niessen L, Islam A. Bangladesh policy on prevention and control of noncommunicable diseases: a policy analysis. BMC Public Health. 2017;17:1-11.

36. Al-Daghri NM, Al-Attas OS, Alokail MS, Alkharfy KM, Yousef M, Sabico SL, et al. Diabetes mellitus type 2 and other chronic non-communicable diseases in the central region, Saudi Arabia (Riyadh cohort 2): a decade of 
an epidemic. BMC Med. 2011;9:1-6.

37. Akl C, Akik C, Ghattas H, Obermeyer CM. Gender disparities in midlife hypertension: a review of the evidence on the Arab region. Women's midlife Heal. 2017;3:1-10.

38. Rozanski A, Blumenthal JA, Kaplan J. Impact of psychological factors on the pathogenesis of cardiovascular disease and implications for therapy. Circulation. 1999;99:2192-217.

39. Scavini M, Stidley CA, Shah VO, Narva AS, Tentori F, Kessler DS, et al. Prevalence of diabetes is higher among female than male Zuni Indians. Diabetes Care. 2003;26:55-60.

40. Barker M, Chorghade G, Crozier S, Leary S, Fall C. Socio-economic factors, lifestyle and gender differences in body mass index in rural India. J Nutr. 2006;136:3062.

41. Agho KE, Osuagwu UL, Ezeh OK, Ghimire PR, Chitekwe S, Ogbo FA. Gender differences in factors associated with prehypertension and hypertension in Nepal: A nationwide survey. PLoS One. 2018;13:e0203278.

42. Oparil S, Acelajado MC, Bakris GL, Berlowitz DR, Cífková R, Dominiczak AF, et al. Hypertension. Nat Rev Dis Prim. 2018;4:18014.

43. Pinto E. Blood pressure and ageing. Postgrad Med J. 2007;83:109-14.

44. Akter J, Shahjahan M, Hossain S, Chowdhury HA, Ahmed KR, Fatema K, et al. Determinants of overweight and obesity among Bangladeshi diabetic women of reproductive age. BMC Res Notes. 2014;7:1-6.

45. Chowdhury MAB, Uddin MJ, Khan HMR, Haque MR. Type 2 diabetes and its correlates among adults in Bangladesh: a population based study. BMC Public Health. 2015;15:1-11.

46. González K, Fuentes J, Márquez JL. Physical inactivity, sedentary behavior and chronic diseases. Korean J Fam Med. 2017;38:111.

47. Abarca-Gómez L, Abdeen ZA, Hamid ZA, Abu-Rmeileh NM, Acosta-Cazares B, Acuin C, et al. Worldwide trends in body-mass index, underweight, overweight, and obesity from 1975 to 2016: a pooled analysis of 2416 population-based measurement studies in 128. 9 million children, adolescents, and adults. Lancet. 2017;390:2627-42.

48. Chowdhury MAB, Adnan MM, Hassan MZ. Trends, prevalence and risk factors of overweight and obesity among women of reproductive age in Bangladesh: a pooled analysis of five national cross-sectional surveys. BMJ Open. 2018;8:e018468.

49. Linderman GC, Lu J, Lu Y, Sun X, Xu W, Nasir K, et al. Association of body mass index with blood pressure among 1.7 million Chinese adults. JAMA Netw open. 2018;1:e181271-e181271.

50. Misra A, Jayawardena R, Anoop S. Obesity in South Asia: phenotype, morbidities, and mitigation. Curr Obes Rep. 2019;8:43-52.

51. Hills AP, Arena R, Khunti K, Yajnik CS, Jayawardena R, Henry CJ, et al. Epidemiology and determinants of type 2 diabetes in south Asia. lancet Diabetes Endocrinol. 2018;6:966-78.

52. Booth FW, Roberts CK, Laye MJ. Lack of exercise is a major cause of chronic diseases. Compr Physiol. 2012;2:1143.

53. American Diabetes Association. Diagnosis and classification of diabetes mellitus. 2010.

54. Talukder A, Hossain MZ. Prevalence of Diabetes Mellitus and Its Associated Factors in Bangladesh: Application of Two-level Logistic Regression Model. Sci Rep. 2020;10:1-7.

55. Brugnara L, Murillo S, Novials A, Rojo-Martínez G, Soriguer F, Goday A, et al. Low physical activity and its association with diabetes and other cardiovascular risk factors: a nationwide, population-based study. PLoS 
One. 2016;11:e0160959.

56. Hu G, Jousilahti P, Barengo NC, Qiao Q, Lakka TA, Tuomilehto J. Physical activity, cardiovascular risk factors, and mortality among Finnish adults with diabetes. Diabetes Care. 2005;28:799-805.

57. Association AD. Physical activity/exercise and diabetes. Diabetes Care. 2004;27 suppl 1:s58-62.

58. Miller YD, Dunstan DW. The effectiveness of physical activity interventions for the treatment of overweight and obesity and type 2 diabetes. J Sci Med Sport. 2004;7:52-9.

59. Hayes C, Kriska A. Role of physical activity in diabetes management and prevention. J Am Diet Assoc. 2008;108:S19-23.

60. Herbst A, Bachran R, Kapellen T, Holl RW. Effects of regular physical activity on control of glycemia in pediatric patients with type 1 diabetes mellitus. Arch Pediatr Adolesc Med. 2006;160:573-7.

61. Tareque MI, Koshio A, Tiedt AD, Hasegawa T. Are the rates of hypertension and diabetes higher in people from lower socioeconomic status in Bangladesh? Results from a nationally representative survey. PLoS One. 2015;10:e0127954.

62. Bangladesh Society of Medicine, World health organization ministry of health and family welfare. Noncommunicable disease risk factor survey Bangladesh 2010. Bangladesh. 2021.

63. Bureau of Statistics. Ministry of planning government of B. Bangladesh Statistics 2017. 2017.

64. Cunningham TJ, Ford ES, Rolle I V, Wheaton AG, Croft JB. Associations of self-reported cigarette smoking with chronic obstructive pulmonary disease and co-morbid chronic conditions in the United States. COPD J Chronic Obstr Pulm Dis. 2015;12:281-91.

65. Wang M, Luo X, Xu S, Liu W, Ding F, Zhang X, et al. Trends in smoking prevalence and implication for chronic diseases in China: serial national cross-sectional surveys from 2003 to 2013. Lancet Respir Med. 2019;7:3545.

66. Flaherty KT, Fuchs CS, Colditz GA, Stampfer MJ, Speizer FE, Willett WC, et al. A prospective study of body mass index, hypertension, and smoking and the risk of renal cell carcinoma (United States). Cancer Causes Control. 2005;16:1099-106.

67. Kannel WB, Higgins M. Smoking and hypertension as predictors of cardiovascular risk in population studies. J Hypertens Suppl Off J Int Soc Hypertens. 1990;8:S3-8.

68. Narkiewicz K, Kjeldsen SE, Hedner T. Is smoking a causative factor of hypertension? 2005.

69. Virdis A, Giannarelli C, Fritsch Neves M, Taddei S, Ghiadoni L. Cigarette smoking and hypertension. Curr Pharm Des. 2010;16:2518-25.

\section{Supplementary Files}

This is a list of supplementary files associated with this preprint. Click to download.

- SupplementaryTable.docx 